\title{
Campylobacter jejuni adapts to aerobic metabolism in the environment
}

\author{
D. M. JONES, E. M. SUTCLIFFE, R. RIOS, A. J. FOX and A. CURRY
}

Public Health Laboratory Service, Withington Hospital, Manchester M20 8LR

\begin{abstract}
Summary. Campylobacter jejuni, when left on blood agar for prolonged periods, was found to survive better in air than under micro-aerobic conditions. After a period of 2-3 days in air, all strains of $C$. jejuni examined grew freely in air on subculture, and could be further subcultured apparently indefinitely in air. This adaptation to aerobic metabolism was accompanied by a change in colony morphology and some changes in outer-membrane protein patterns, but no change in serotyping reactions. The ability to colonise mice was unaltered as was the helical morphology of growing cells. The important survival phase of $C$. jejuni, when outside the animal gut, involves not only a change to coccal morphology but also fundamental changes in the metabolism of the organism. These changes are likely to be relevant to techniques required for culturing $C$. jejuni from foods and environmental sources.
\end{abstract}

\section{Introduction}

It is the established view that the thermophilic campylobacters are strictly micro-aerophilic ${ }^{1}$ and require $5-10 \%$ oxygen for satisfactory growth. This is in keeping with the conditions that prevail in the animal gut, which is the natural habitat of these organisms. Campylobacters cannot multiply in the environment and for continued existence these organisms may have to survive in conditions that are very different from those in the gut. It seems logical that modifications observed to occur to these bacteria in the environment, such as the development of coccal forms, ${ }^{2}$ will also be accompanied by changes in metabolic activities. A probable change would be the ability to survive in nonmicro-aerobic conditions.

To test this proposition, we studied the survival of Campylobacter jejuni on solid laboratory media at various temperatures and compared survival in microaerobic conditions and in air.

\section{Materials and methods}

The medium used for growing campylobacters was Blood Agar Base No. 2 (Oxoid) with difibrinated horse blood $5 \%$. A conventional micro-aerobic atmosphere was attained by incubating plates, in jars containing Gas Generating Kit (Anaerobic System Code BR38, Oxoid) without catalysts. Humidity was maintained for plates under aerobic conditions by placing them in jars with open valves and including a container of water in each jar. Plates in aerobic culture were opened and ventilated each day to ensure continuous aerobic conditions.

\section{Cultures}

Strain 13997, used for quantitative survival experiments, was $C$. jejuni serotype Lior 1 Penner 4 isolated from a clinical human infection. Mouse colonisation experiments were done with aerobically adapted strains of $C$. jejuni 5813 (Lior 1 Pen 4) and C. jejuni Pen 10 (MK1, supplied by Dr J. Penner). The mouse colonising dose for these strains after conventional micro-aerobic culture had been ascertained previously. ${ }^{2}$ Other $C$. jejuni cultures were recent human clinical isolates submitted to the laboratory for serotyping.

\section{Quantitative estimates of survival}

Strain 13997 was incubated on blood agar in a micro-aerobic atmosphere at $37^{\circ} \mathrm{C}$ for $48 \mathrm{~h}$. A suspension (MacFarlanes No. 1) of this culture in nutrient broth was diluted to $10^{-6}$ and $0.02 \mathrm{ml}$ of this dilution was spread on to each of 100 blood agar plates. These were then incubated micro-aerobically at $42^{\circ} \mathrm{C}$ for $18 \mathrm{~h}$. At this stage it was possible to count the colonies established on each plate. The average number was 28 and plates deviating from this number by more than two colonies were discarded. Half the plates were then incubated in air and the remainder in a micro-aerobic atmosphere at $42^{\circ} \mathrm{C}$. The total number of viable organisms/plate was found by suspending the total growth in $5 \mathrm{ml}$ of nutrient broth and making serial 10fold dilutions; surface viable counts were made on well-dried blood agar plates incubated at $37^{\circ} \mathrm{C}$ for $48 \mathrm{~h}$ in a micro-aerobic atmosphere. The total number of viable cells/plate was estimated after the initial microaerobic incubation for $18 \mathrm{~h}$ (Day 0), and thereafter daily for 6 days and then less frequently for up to 4 5 weeks, for both series of plates. The experiment was 
repeated with aerobic and micro-aerobic plates incubated at $37^{\circ} \mathrm{C}$ and a similar series of plates was held at $4^{\circ} \mathrm{C}$ for estimation of low temperature survival.

\section{Cell morphology}

At various stages during the induction of aerotolerance the morphology of the organisms was studied by the examination of gram-stained films and by electronmicroscopy. A freshly made suspension of growth in distilled water was placed on a pioloformcoated EM grid, any excess was removed and the preparations were then stained with phosphotungstic acid (pH 6.5) 3\%. The grids were examined in a Philips EM420 electronmicroscope.

\section{Investigation of aerotolerance}

Twenty-five recently isolated and serotyped strains of $C$. jejuni were used. Cultures were grown microaerobically for $18 \mathrm{~h}$ at $42^{\circ} \mathrm{C}$ on blood agar, thereafter the plates were incubated in air. Subcultures were made on to blood agar after 2, 3 and 4 days and also incubated in air. These growths were then serially subcultured and after 15-20 subcultures in air were again serotyped.

The outer-membrane protein profiles of the established aerotolerant forms of three of these strains were compared with their original micro-aerophilic forms.

\section{Outer-membrane protein profiles}

Air-adapted and non-adapted organisms were grown on blood agar under aerobic and micro-aerobic conditions respectively, and harvested from 10 plates grown for $48 \mathrm{~h}$ at $42^{\circ} \mathrm{C}$. The growth from the plates was scraped into a solution containing sodium deoxycholate $0.5 \% \mathrm{w} / \mathrm{v}$ in $0.1 \mathrm{M}$ Tris- $\mathrm{HCl}, \mathrm{pH} 8.5,0.01 \mathrm{M}$ EDTA. Organisms were shaken $(300 \mathrm{rpm})$ in the presence of glass beads at $56^{\circ} \mathrm{C}$ for $1 \mathrm{~h}$. Membranes were recovered by differential centrifugation and resuspended in de-ionised double distilled water at a protein concentration of $1 \mathrm{mg} / \mathrm{ml}$ with phenyl methyl sulphonyl fluoride $40 \mu \mathrm{g} / \mathrm{ml}$.

\section{$S D S-P A G E$}

Outer-membrane preparations were solubilised in buffer containing SDS $1.6 \% \mathrm{v} / \mathrm{v}, 0.5 \mathrm{M} 2$-mercaptoethanol in $0.05 \mathrm{M}$ Tris- $\mathrm{HCl}, \mathrm{pH} \mathrm{6.5}$. Proteins were separated in acrylamide $12 \%$ slab gels with acrylamide $3 \%$ stacking gels. Outer-membrane protein (OMP) profiles were visualised by staining with Coomassie Blue.

\section{Mouse colonisation}

Two strains of $C$. jejuni previously used in mouse colonisation experiments, were adapted to aerobic metabolism and a suspension of organisms grown in air were fed to day-old mice in estimated doses of $10-10^{3} \mathrm{cfu} / 0.02 \mathrm{ml}$. Precise surface viable counts were performed on the suspensions fed to the mice. The mice were killed after 1 week and direct cultures were made from a $1-\mathrm{cm}$ portion of lower intestine on to Preston Agar (Modified CCDA-Preston, Oxoid) incubated at $37^{\circ} \mathrm{C}$ both in air and in a micro-aerobic atmosphere. A similar portion of lower intestine was placed in Preston Enrichment Broth ${ }^{3}$ and this was subcultured after $48 \mathrm{~h}$, the plates were incubated at $37^{\circ} \mathrm{C}$ in air and in micro-aerobic conditions.

\section{Results}

\section{Quantitative estimates of survival}

In survival experiments on blood agar at $42^{\circ} \mathrm{C}$ the total viable counts/plate were initially greater for the first 3 days on the micro-aerobically than the aerobically maintained plates (fig. 1). By the fourth day this difference had disappeared and subsequently the viable counts remained higher on the plates maintained in air. After 14 days, organisms could no longer be cultured from the micro-aerobic plates whereas viable organisms could be recovered from the aerobic plates for $>3$ weeks. The sequence of events when the plates were kept at $37^{\circ} \mathrm{C}$ and $4^{\circ} \mathrm{C}$ was essentially similar, but survival was more prolonged (figs 2 and 3 ). The aerobic plates again showed an initial greater fall in viable counts but after $c$. 8-10 days there was little difference, and after this the aerobic plates showed significantly greater survival. In addition, at $4^{\circ} \mathrm{C}$ plates with air-adapted strain 13997 were included (see below). The strain was grown overnight in air and the plates were then placed in the refrigerator. The total viable counts showed little fall over a 34-day observation period (fig. 3), indicating better survival if the culture was already air-adapted when placed at $4^{\circ} \mathrm{C}$.

\section{Cellular morphology}

In the first 4 days on both the aerobic and microaerobic series of plates, the organisms became predominantly coccal in morphology (fig. 4). Gramstained films made later showed mainly poorly staining non-viable cocci, a small proportion of well-stained cocci, presumably viable, and occasional long filamentous forms.

\section{Aerotolerance}

It was observed that subcultures on blood agar taken from the plates maintained in air grew normally under micro-aerobic conditions, but, additionally, grew equally well when incubated in air. Moreover, these subcultures could be continuously passaged on blood agar incubated in air at either $42^{\circ} \mathrm{C}$ or $37^{\circ} \mathrm{C}$. To establish whether this was a characteristic of $C$. jejuni 


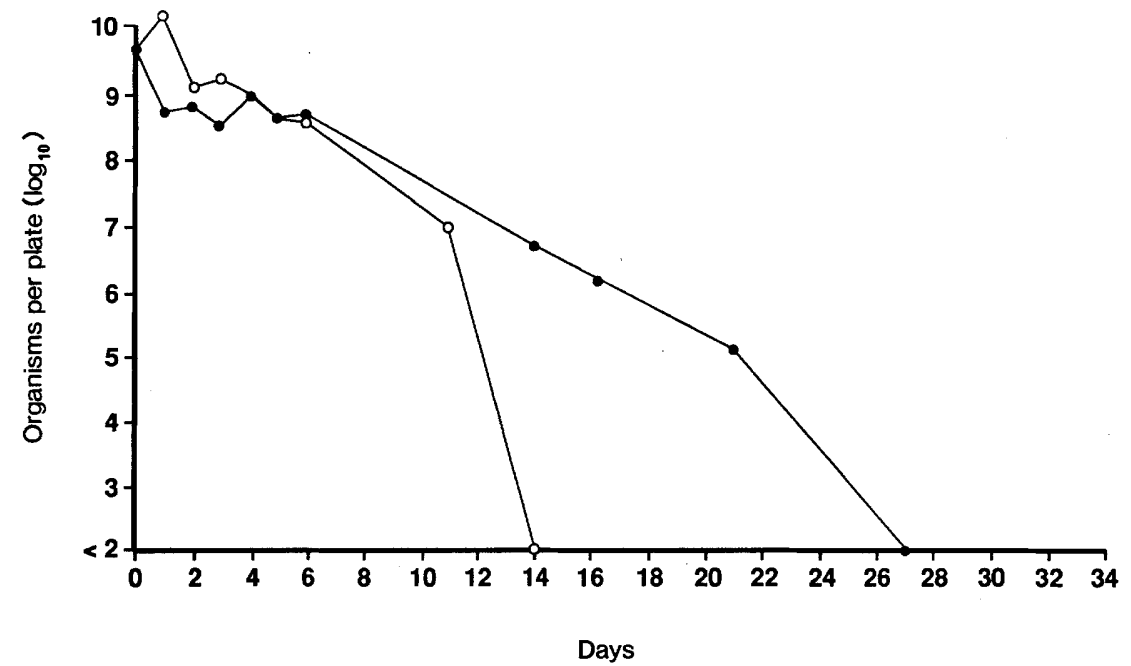

Fig. 1. Total viable counts of $C$. jejuni 13997 surviving on blood agar plates held at $42^{\circ} \mathrm{C}$ in micro-aerobic conditions $(-\mathrm{O}-)$ and in air $(-\bullet)$.

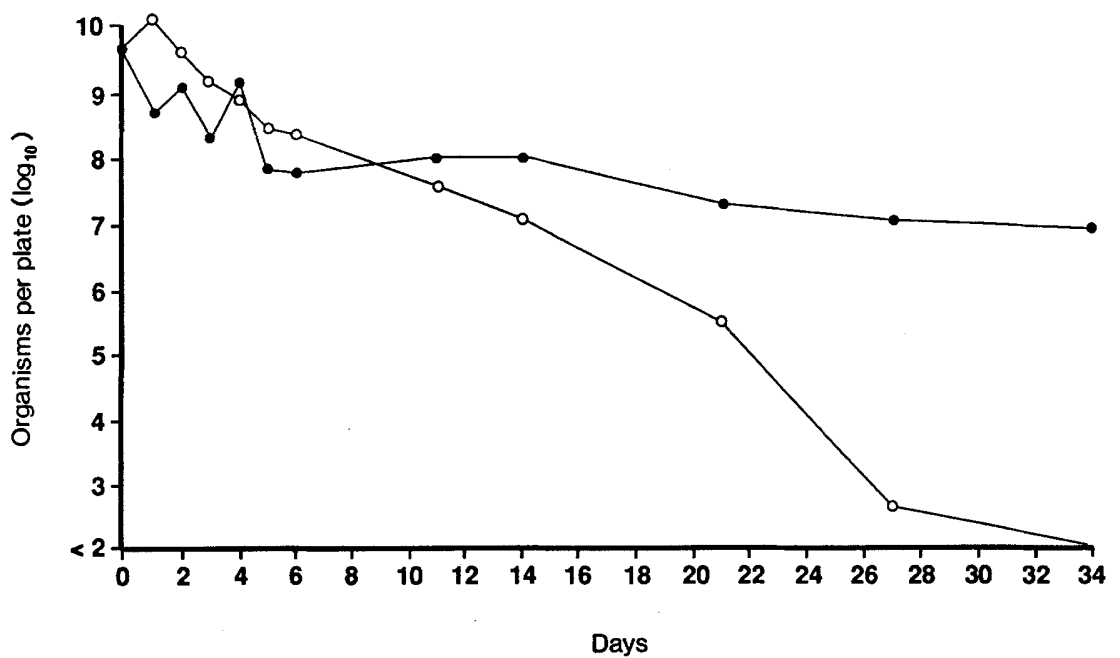

Fig. 2. Total viable counts of $C$. jejuni 13997 surviving on blood agar plates held at $37^{\circ} \mathrm{C}$ in micro-aerobic conditions (-O -$)$ and in air $(-\bullet)$.

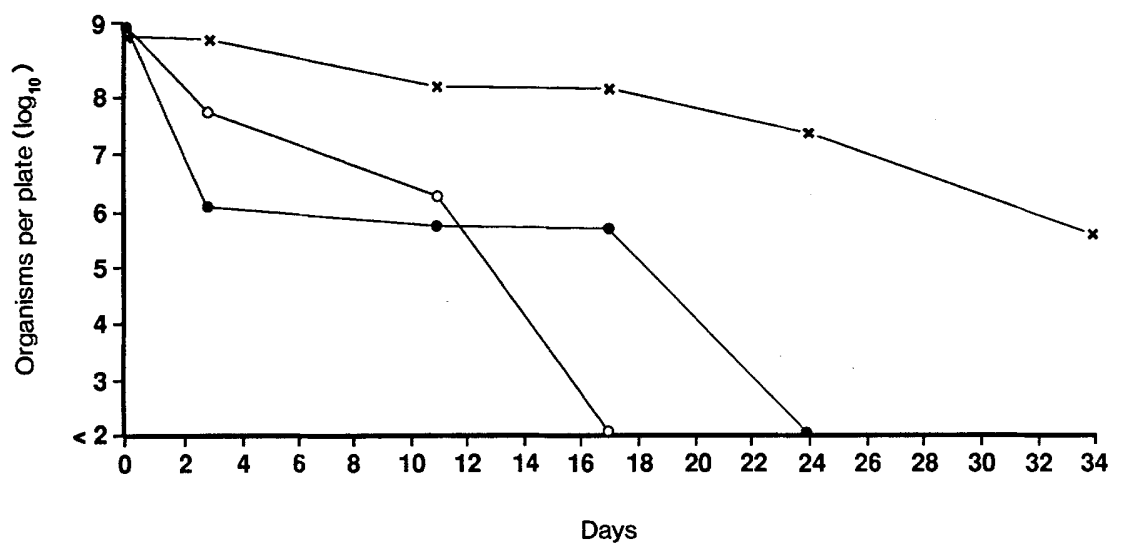

Fig. 3. Total viable counts of $C$. jejuni 13997 surviving on blood agar plates held at $4^{\circ} \mathrm{C}$ in micro-aerobic conditions $(-\mathrm{O}-)$ and in air (- -). Viable counts of C. jejuni 13997 (air-adapted) on blood agar at $4^{\circ} \mathrm{C}$ in air $(-\mathbf{x}-$ ).

13997 or a general phenomenon, we treated 25 recent isolates in a similar way. These strains were first grown for $18 \mathrm{~h}$ on blood agar under micro-aerobic conditions and then further incubated in air. Subcultures taken 2-4 days later always proved to be capable of growing in air, as well as micro-aerobically. These 25 strains were subcultured for 20 passages in air after which they were re-serotyped. Adaptation to stable aerobic metabolism did not affect the serotyping reactions of either of the Lior (heat-labile) or Penner (heat-stable) systems.

\section{Colonial morphology}

The subcultures that were air-adapted showed a change in colonial morphology. The colonies were 


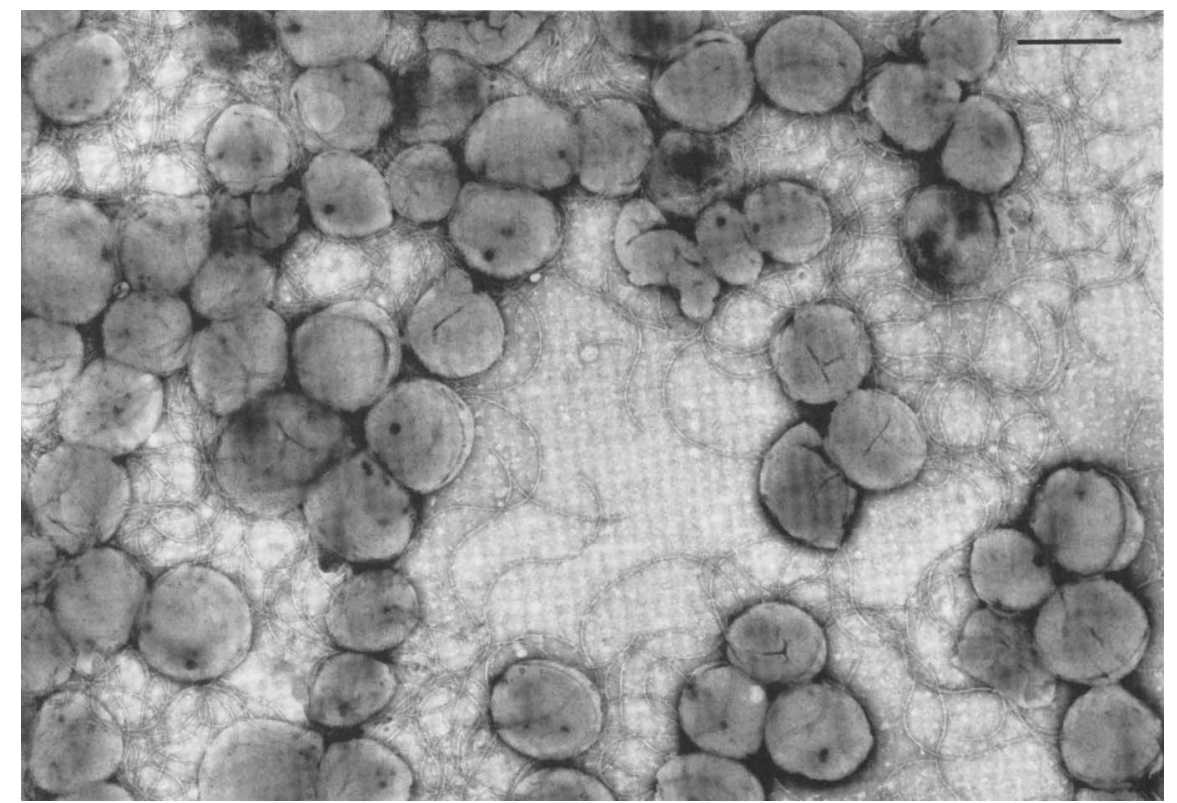

Fig. 4. Electronmicrograph of negatively-stained C. jejuni after overnight growth at $37^{\circ} \mathrm{C}$ in a micro-aerobic atmosphere followed by incubation for $24 \mathrm{~h}$ in air. Bar $=1000 \mathrm{~nm}$.

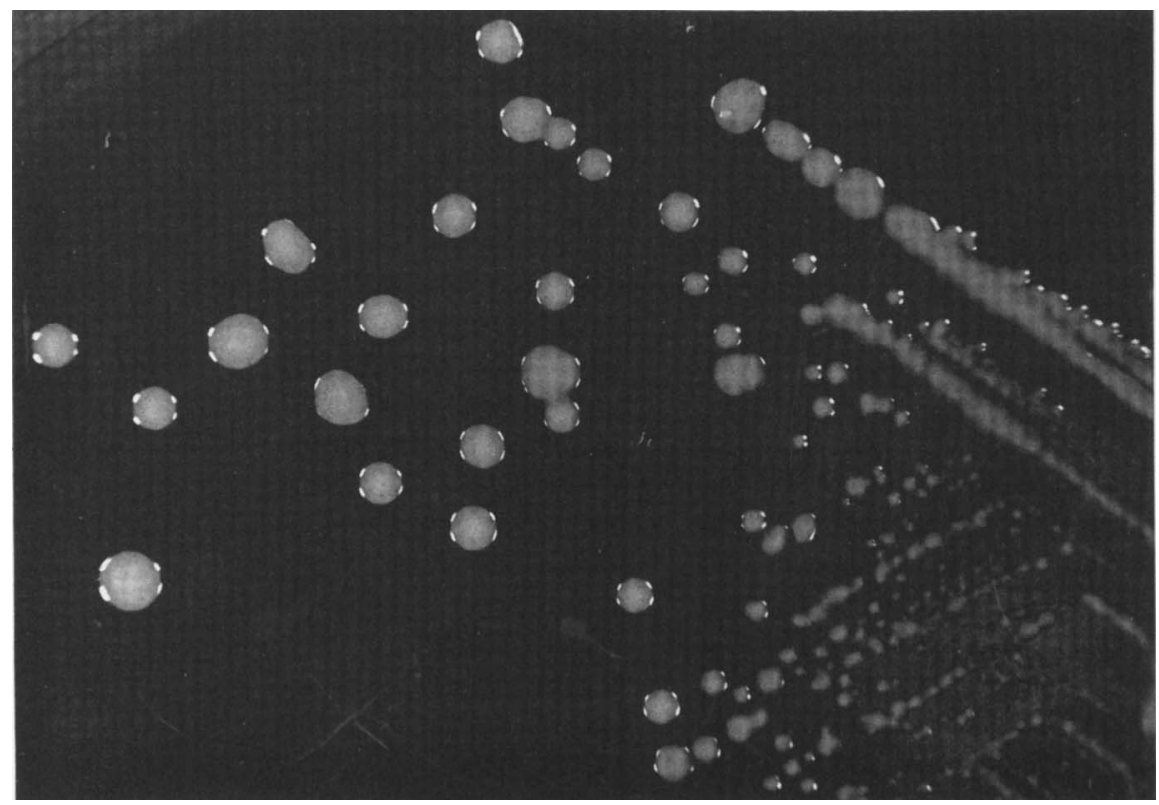

Fig. 5. Air-adapted C. jejuni growing overnight on blood agar in air

opaque and convex with a sharply defined margin rather than the effuse colony form of the original micro-aerophilic strain (fig. 5). The morphology of individual cells from these two types of colony was indistinguishable, they were flagellate and vibrioid, typical of campylobacters. Strains of $C$. jejuni in the stable aerobic form were readily reconverted to both micro-aerobic metabolism and to original colonial morphology by single subculture in micro-aerobic conditions.

\section{Outer-membrane protein profiles}

OMP profiles of three strains of $C$.jejuni $(\mathrm{Ll}, \mathrm{P} 4,50$; L2. P1; L8, P11) after micro-aerobic growth and adaptation to growth in air, were examined by SDSPAGE. Several differences in the peptide composition of OMP of micro-aerophilic and aerotolerant organisms prepared by deoxycholate extraction were observed (fig. 6). With the protein stains used, peptide bands in the range $45-50$ and $30-35 \mathrm{kDa}$ were absent or reduced beyond detection from OMP preparations from organisms after aerobic culture. Differential expression of high-mol.-wt components $(100$ $110 \mathrm{kDa}$ ) was also apparent following growth in the different atmospheric conditions.

\section{Mouse colonisation}

The stable aerobic forms of $C$. jejuni 5813 colonised suckling mice at a dose of $>18 \mathrm{cfu}$ (table). This is not different from the colonising ability of this strain in the conventional micro-aerophilic form. When $2 \times 10^{4} \mathrm{cfu}$ aerobic forms of $C$. jejuni Pen 10 were fed to day-old 


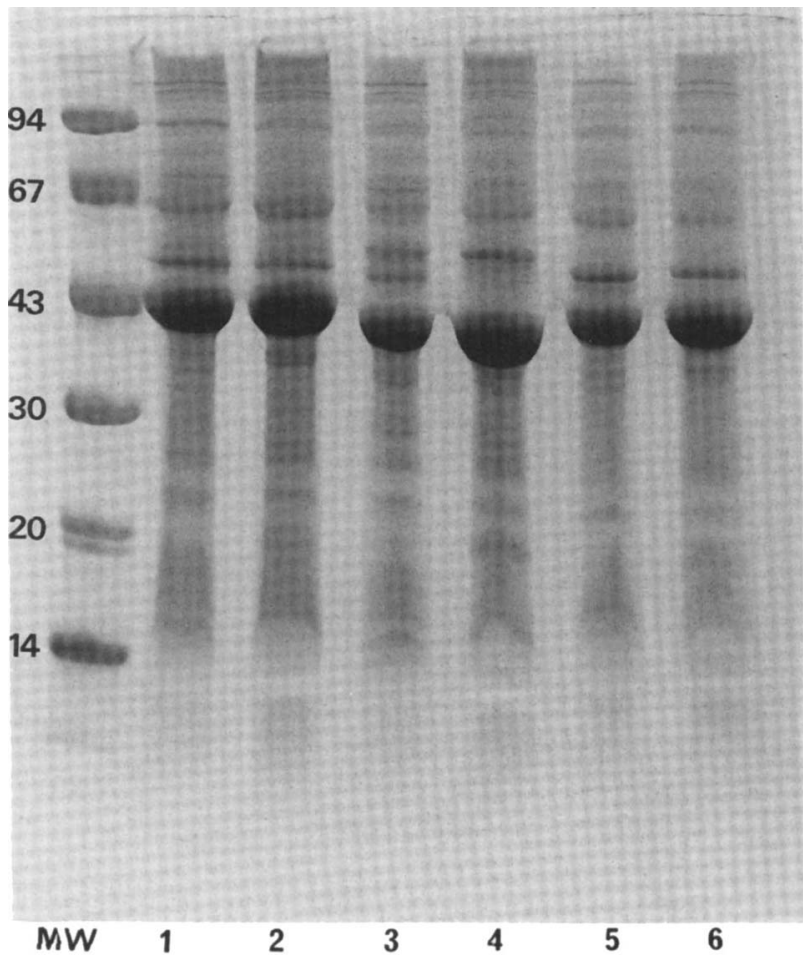

Fig. 6. Outer-membrane proteins of micro-aerobic and aerobic cultures of three strains of $C$. jejuni: serotype L1 P4 micro-aerobic (lane 1) and aerobic (2); serotype L2 P1 micro-aerobic (3) and aerobic (4); L8 P11 micro-aerobic (5) and aerobic (6).

Table. Colonising ability of oxygen-adapted strains in dayold mice

\begin{tabular}{lccc}
\hline $\begin{array}{c}\text { Strain } \\
\text { no. }\end{array}$ & $\begin{array}{c}\text { Dose (cfu) } \\
\text { or organisms }\end{array}$ & $\begin{array}{c}\text { Number of mice } \\
\text { challenged }\end{array}$ & $\begin{array}{c}\text { Number of mice } \\
\text { colonised }\end{array}$ \\
\hline 5813 & $1.8 \times 10^{3}$ & 5 & 5 \\
5813 & $1.8 \times 10^{2}$ & 6 & 6 \\
5813 & 18 & 6 & 3 \\
Pen 10 & $2 \times 10^{4}$ & 6 & 2 \\
\hline
\end{tabular}

mice, two of six mice were colonised. The colonising dose for micro-aerophilic Pen 10 organisms was estimated at $6 \times 10^{4} \mathrm{cfu}$.

In direct cultures from mouse intestine, strains grew equally well when incubated either in air or a microaerobic atmosphere and continued to show the differences in colonial morphology. Subcultures of the enrichment culture of mouse intestine in Preston broth also grew equally well in either atmosphere.

\section{Discussion}

In studies of the survival of $C$. jejuni in pond water, we previously observed that after organisms are no longer recoverable by culture, they still survive in a state that can colonise mice. ${ }^{2}$ The animal gut is capable of reviving the dormant organism whereas conventional laboratory media do not. It is likely that changes in metabolism occur in the dormant bacterial cell, and that until these are better understood the appropriate methods for culturing dormant cells will not be developed. In the experiments reported here we looked at the survival of $C$. jejuni in a situation where nutrients remain available at least to some degree, and at higher temperatures growth and division are possible at the same time as degeneration. The first observation was that at $42^{\circ} \mathrm{C}, 37^{\circ} \mathrm{C}$ and $4^{\circ} \mathrm{C}$ survival was more prolonged in air than in a micro-aerobic atmosphere. The overall survival at $42^{\circ} \mathrm{C}$ (where growth might occur) and at $4^{\circ} \mathrm{C}$ (where it would not) were closely similar, survival being most prolonged at $37^{\circ} \mathrm{C}$. The second important observation was that if subcultures were taken from plates kept in air, the organisms behaved as conventional aerobes and could be continuously maintained by subculture in air, provided that humidity was adequate. This was clear evidence that during the survival process the metabolic processes change so that $C$. jejuni survives preferentially and can even grow in $21 \%$ oxygen.

Although adaptation to aerobic metabolism was accompanied by change in colony morphology and some change in the OMP profile, the individual cell morphology was unchanged, as was the ability to colonise mice and the serotype of the strain. Obviously the ability of dormant campylobacters to re-colonise animals is essential for the continued survival of the species, so the finding that this property was unchanged is as expected.

At $4^{\circ} \mathrm{C}$ there is less metabolic energy available and the adaptation to air may have occurred more slowly (fig. 3). However, an air-adapted strain clearly outperformed all others at $4^{\circ} \mathrm{C}$ in terms of survival. This result reinforces the observation that the air-adapted form is also particularly adapted for survival.

The cultural characteristics of the air-adapted strains recovered from the mouse gut were of interest. The isolation of these strains from gut contents by direct culture in air indicated that they were able to retain the propensity for growth in air at least over the short period of gut colonisation in these studies. Moreover, culture of gut contents in enrichment broth did not affect the ability for prompt growth in air on further subculture on solid media. These observations indicate that the changes that take place in the metabolic pathways of the organism to enable aerobic growth have some measure of durability in other than aerobic environments. Aerotolerant, non-thermophilic campylobacter-like organisms (Arcobacter butzleri $)^{4}$ are clearly different from $C$. jejuni, but these organisms may have metabolic similarities to airadapted C. jejuni.

In the dormant survival phase of $C$. jejuni the change to coccal morphology is rapid and obvious. Furthermore, it is clear that fundamental metabolic changes in the organisms also occur and these will require further study. It is possible that the existence of the viable but non-culturable phase for campylobacters is only an expression of our lack of under- 
standing of how fundamentally the metabolic requirements of the organism change when it is in the environment. The content of the media and the atmospheres used for incubation, designed for iso-

\section{References}

1. Skirrow MB. Campylobacter, Helicobacter and other mobile curved gram-negative rods. In: Parker MT, Collier LH (eds) Topley and Wilson's Principles of bacteriology, virology and immunity, 8th edn. London, Arnold. 1990:2: 531.

2. Jones DM, Sutcliffe EM, Curry A. Recovery of viable but nonculturable Campylobacter jejuni. J Gen Microbiol 1991; 137: $2477-2482$. lating micro-aerophilic organisms from the gut, may be quite inappropriate for rousing these altered organisms from dormancy, and further work is required to define more appropriate cultural conditions.

3. Bolton FJ, Coates D, Hinchcliffe PM, Robertson L. Comparison of selective media for the isolation of Campylobacter jejuni/coli. J Clin Pathol 1983; 36: 78-83.

4. Taylor DN, Kiehlbauch JA, Tee W, Pitarangsi C, Echeverria P. Isolation of Group 2 Aerotolerant Campylobacter species from Thai children with diarrhoea. $J$ Infect Dis 1991; 163: 1062-1067. 